\title{
Mean Platelet Volume to Lymphocyte Ratio as a Novel Marker for Diabetic Nephropathy
}

\author{
Mehmet Zahid Kocak, Gulali Aktas, Edip Erkus, Tuba T. Duman, Burcin M. Atak and Haluk Savli
}

\begin{abstract}
Objective: To compare mean platelet volume to lymphocyte ratio (MPVLR) of diabetic nephropathy subjects to those diabetics without diabetic nephropathy.

Study Design: Observational, cross-sectional study.

Place and Duration of Study: Tertiary referral hospital, in Bolu, Turkey, from July to December 2017.

Methodology: Patients with type 2 diabetes mellitus admitted to the Internal Medicine Clinic, were included. Patients were divided into two groups according to the urinary microalbumin/creatinine ratio: diabetic nephropathy and non-nephropathy group. MPVLR was calculated mathematically by division of MPV by lymphocyte count.

Results: There were 162 subjects ( 79 females, 83 males). The MPVLR in patients with diabetic nephropathy and nonnephropathic diabetic groups were $4.1(2.09-11.84)$ and 3.4 (1.37-25.56), respectively. The difference was reached statistically significant level $(p<0.001$ ). The best cut-off value for MPVLR was 3.66 (AUC=0.733, $p<0.001$ ); MPVLR predicted diabetic nephropathy with $71.1 \%$ sensitivity and $67.4 \%$ specificity, at this level.

Conclusion: The MPVLR is an easily calculated and efficient index that can be considered a powerful and independent predictor of diabetic nephropathy in diabetic patients. We suggest that, it can be useful adjunct to standard tests in the diagnosis of diabetic nephropathy.
\end{abstract}

Key Words: Diabetic nephropathy, Inflammation, Mean platelet volume to lymphocyte ratio.

\section{INTRODUCTION}

Inflammation has been proposed as a cardinal pathogenetic mechanism in diabetic nephropathy in experimental and clinical studies.1,2 Acute phase reactants and various cytokines are elevated in patients with diabetic nephropathy. ${ }^{3-5}$

Mean platelet volume (MPV) is an index of platelet size and activity. ${ }^{6}$ As a haemogram derived inflammatory marker, platelet to lymphocyte ratio (PLR), was studied in various cancers, $, 7,8$ chronic renal failures, ${ }^{9}$ and coronary artery diseases. ${ }^{10}$ In addition, an increase in PLR was found to be associated with increased microalbuminuria due to increased inflammation and endothelial dysfunction. 11

Recent studies have suggested that MPV/lymphocyte ratio (MPVLR) may be a predictor of short and long term prognosis in diabetic patients with myocardial infarction. ${ }^{11,12}$ The relation between MPVLR and diabetic nephropathy is based on the hypothesis that the size of the platelets demonstrates platelet activity more accurately than platelet count, ${ }^{13}$ and the use of MPV in place of platelet counts in PLR. To the authors'

Department of Internal Medicine, Abant Izzet Baysal (AIB)

University Hospital, Bolu, Turkey

Correspondence: Dr. Mehmet Zahid Kocak, Department of Internal Medicine, Abant Izzet Baysal (AIB) University Hospital, Bolu, Turkey

E-mail:mehmetzahidkocak@hotmail.com

Received: January 15, 2018; Accepted: July 18, 2018 knowledge, this is the first study of MPVLR in relation to diabetic nephropathy.

The objective of the study was to compare mean platelet volume to lymphocyte ratio (MPVLR) of diabetic nephropathy subjects to those diabetics without diabetic nephropathy.

\section{METHODOLOGY}

This descriptive study was performed after the approval of the institutional authority of the university hospital, where the study was conducted. Patients with type 2 diabetes mellitus, who were admitted to the Internal Medicine Clinic between July and December 2017, were included in the study. Patients were divided into two groups according to the urinary microalbumin/creatinine ratio: diabetic nephropathy and non-nephropathy group. Patients' age, gender, systolic and diastolic blood pressures (SBP, DBP), waist circumference, duration of diabetes, and body mass index (BMI) were recorded. The hemoglobin $(\mathrm{Hb})$, hematocrit $(\mathrm{Htc})$, lymphocyte, platelet, MPV, creatinine, fasting blood glucose (FBG), high density lipoprotein (HDL), total cholesterol, triglyceride, low-density protein (LDL), glycated hemoglobin $(\mathrm{HbA} 1 \mathrm{c})$, creatinine and microalbumin in spot urine had been recorded separately and the data was collected retrospectively. MPVLR was calculated mathematically by division of MPV by lymphocyte count. The microalbuminuria was calculated using formula [spot urine microalbumin (gr/l)/spot urine creatinine $\mathrm{g} / \mathrm{dl})] \times 100$. According to this, the patients were divided into 2 groups with and without microalbuminuria. 
Statistical analysis of the data was performed using the IBM Statistics 15.0 (SPSS) statistical package programme. Descriptive statistics were presented as mean $\pm S D$ and median (IQR). Normal distribution of continuous variables was evaluated by KolmogorovSmirnov tests. The Mann-Whitney U-test was used to evaluate the difference between the median of the nonnormally distributed data of two groups. The t-test was used to assess the difference between the means of normal distributed data. Receiver-operating characteristic (ROC) curve analyses were performed to determine MPVLR (PLR and MPV for comparison) cut-off values, area under the curve (AUC), sensitivity, and specificity to predict diabetic nephropathy. Correlation analysis conducted by Pearson correlation test. Statistical significance was accepted as $p<0.05$.

\section{RESULTS}

A total of 162 subjects ( 79 females and 83 males) were enrolled in the study. There were $76(47 \%)$ patients in diabetic nephropathy and 86 (53\%) in non-nephropathy group. The mean age of the patients in diabetic nephropathy and non-nephropathy groups were 61.4 \pm 8.6 and $56.7 \pm 9.9$ years, respectively.

The median disease duration was $10(10)$ years in diabetic nephropathy and 5 (8) years in non-nephropathic diabetic group $(p<0.001)$. Serum creatinine level was $0.87(0.36)$ in diabetic nephropathy and $0.8(0.19) \mathrm{mg} / \mathrm{dl}$ in non-nephropathy group $(p=0.06)$. FBG was 197.5 (112) $\mathrm{mg} / \mathrm{dl}$ in the diabetic nephropathy group and 131 (58) $\mathrm{mg} / \mathrm{dl}$ in the non-nephropathy group. In diabetic nephropathy group, FBG was statistically higher than non-nephropathy group $(p<0.001)$. The median value of $\mathrm{HbA1c}$ in the group with diabetic nephropathy was 9.1 (1.9), and 6.8 (1) in the non-nephropathy group, and the difference was statistically significant $(p<0.001)$. Triglyceride levels of diabetic nephropathy and nonnephropathic diabetic groups were $174.5 \mathrm{mg} / \mathrm{dl}$ (112) and $152.5 \mathrm{mg} / \mathrm{dl}$ (89), respectively. The difference was statistically significant $(p=0.034)$. The MPV $(p<0.001)$ was significantly higher in diabetic nephropathy group compared to non-nephropathic diabetic subjects. The MPVLR in patients with diabetic nephropathy and non-nephropathic diabetic groups were 4.1 (1.58) and 3.4 (0.97), respectively. The difference was reached statistically significant level $(p<0.001)$. There were no significant difference among SBP, DBP, WC, BMI, LDL, $\mathrm{HDL}$, total cholesterol levels between study groups ( $p>0.05$ for all). Laboratory data and general characteristics of the study population are shown in Table I.

A ROC curve analysis was used to determine platelet activity parameters (MPVLR, PLR, MPV) in detecting diabetic nephropathy (Figure 1). The best cut-off value for MPVLR was 3.66 (AUC=0.733, $p<0.001$ ), for MPV was 7.73 (AUC=0.697, $p<0.001$ ) and for PLR was 112 (AUC=0.581, $p=0.075$ ). According to ROC analysis, MPVLR predicted diabetic nephropathy with $71.1 \%$ sensitivity and $67.4 \%$ specificity, at 3.66 level. Moreover, it was more sensitive and specific than MPV $(69.7 \%$ sensitivity and $66.6 \%$ specificity) and PLR $(60.5 \%$ sensitivity and $54.7 \%$ specificity) in selecting patients with diabetic nephropathy.

Table I: Demographic characteristics and laboratory parameters of the study groups.

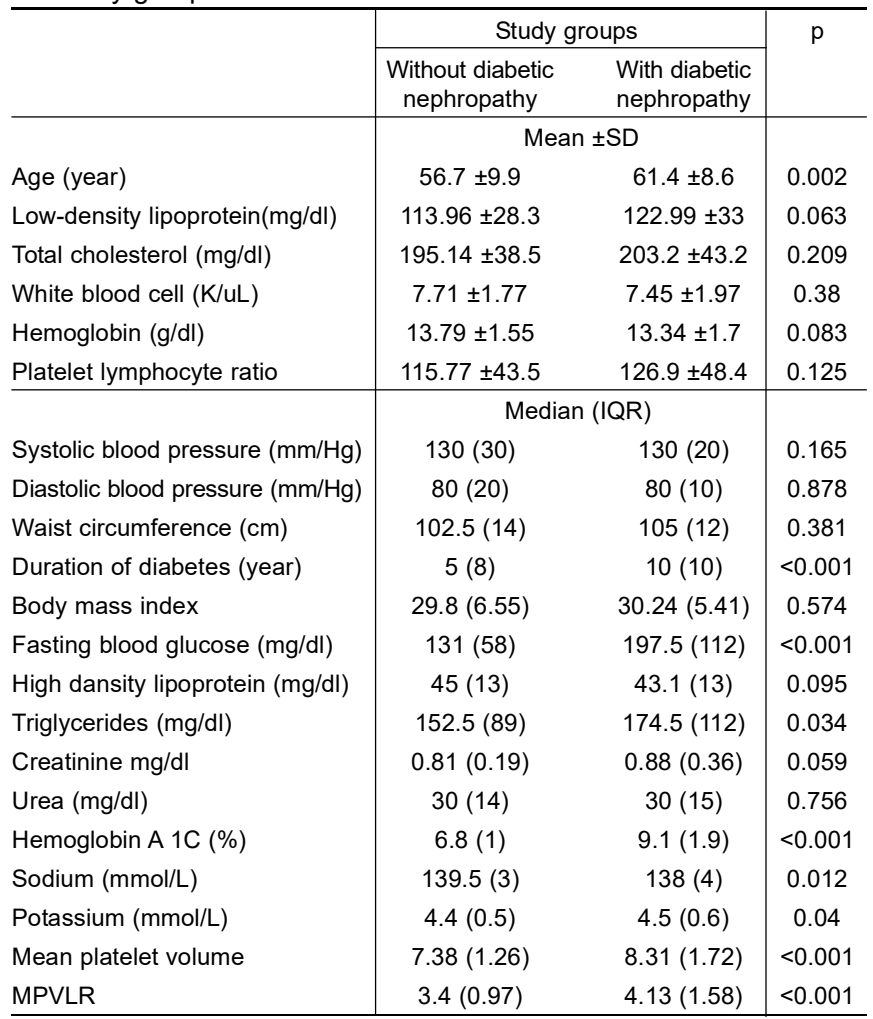

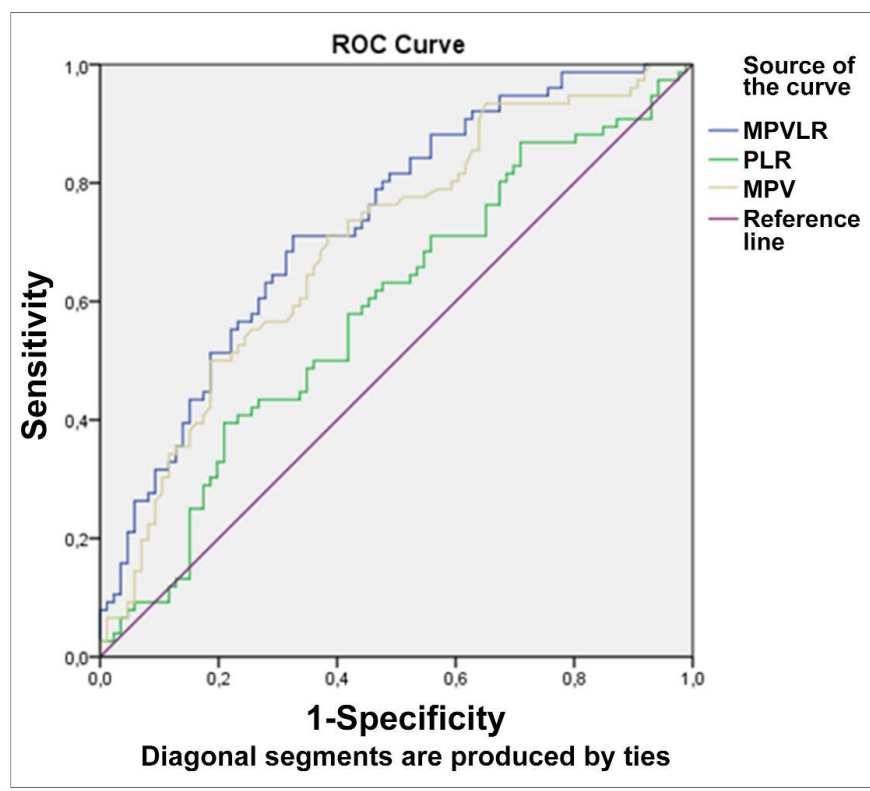

Figure 1: The ROC curves of MPV, PLR, and MPVLR for diabetic nephropathy 


\section{DISCUSSION}

It was demonstrated in this study that presence of diabetic nephropathy can also be detected by MPVLR as well as by microalbuminuria. Moreover, MPVLR was more specific and sensitive than MPV and PLR in selecting diabetic nephropathy subjects. MPVLR levels higher than 3.66 was $71.1 \%$ sensitive and $68 \%$ specific in predicting microalbuminuria in type 2 diabetic patients.

Lymphocytes are essential for a complete inflammatory response, and decreasing lymphocyte counts by induced apoptosis may increase inflammatory damage. 14,15 PLR has been reported to exhibit hyperactivity of the inflammatory pathways. ${ }^{16}$ High platelet counts reflect underlying inflammation, because many inflammatory mediators stimulate megakaryocyte proliferation and lead to relative thrombocytosis. ${ }^{16}$ However, the platelet size shows platelet activity more accurately than the platelet count. ${ }^{13}$ MPVLR, which was calculated using MPV instead of the platelet count in PLR, was claimed to be a more plausible index of platelet activity. ${ }^{13}$ Increased MPVLR levels at the time of admission in diabetic subjects with ST elevation myocardial infarction have been reported to predict short and long-term poor prognosis. ${ }^{12}$

Diabetic nephropathy is a chronic complication that characterised with increased urinary excretion of albumin. ${ }^{17}$ Diabetic nephropathy occurs as a result of the interaction between both genetic and environmental factors. Hyperglycemia, hypertension and genetic predisposition are the main risk factors. ${ }^{18}$ The pathogenesis of microvascular complications of diabetes mellitus has not yet been fully understood. However, hyperglycemia always acts as an initiator and maintenance factor to constantly damage target tissues and organs. In accordance with the literature, present study revealed that the rate of diabetic nephropathy was significantly increased in patients with higher $\mathrm{HbA} 1 \mathrm{c}$ and FBG levels. Authors have studied different markers to predict diabetic nephropathy in literature.19 In the present report, it was found that MPVLR and MVP can both predict diabetic nephropathy.

Podocyte damage occurs at a relatively early stage in chronic renal failure. 20 It has been reported that podocyte damage is typically associated with prominent proteinuria in diabetic nephropathy. 21 Acute phase reactants and various cytokines are elevated in diabetic subjects with nephropathy. ${ }^{3-5}$ Thus, inflammation was supposed as a cardinal pathogenetic mechanism of diabetic nephropathy in experimental and clinical studies.1,2 There are many reports in literature pointed the association between inflammation and diabetic nephropathy. A study expressed that overproduction of inflammatory mediators promote and enhance diabetic nephropathy. 22 Authors found that continuous and lowgrade inflammation and activation of the immune cells are involved in the pathogenesis of diabetes and its microvascular complications, including diabetic nephropathy. ${ }^{23}$ Moreover, Lim et al. suggested that reducing inflammation in diabetic nephropathy could be a promising therapeutic option in the treatment of the disease. 5 Similar to the literature, relationship between diabetic nephropathy and MPVLR was disclosed in present report, since MPVLR was significantly higher in patients with diabetic nephropathy as compared to those diabetics without nephropathy.

The limitations of this study are: relatively small study cohort and retrospective design, which make the present results weaker. However, to the authors' knowledge, this is the first study in literature that demonstrated an association between MPVLR and diabetic nephropathy. It is believed that further studies will be conducted by following the present report.

\section{CONCLUSION}

MPVLR is an easily calculated and efficient index that can be considered a powerful and independent predictor of diabetic nephropathy in diabetic patients. The authors suggest that, it can be useful adjunct to standard tests in the diagnosis of diabetic nephropathy.

\section{REFERENCES}

1. Dalla Vestra M, Masiero A, Roiter AM, Saller A, Crepaldi G, Fioretto $P$. Is podocyte injury relevant in diabetic nephropathy? Diabetes 2003; 52:1031-5.

2. Chow F, Nikolic-Paterson D, Ma F, Ozols E, Rollins B, Tesch G. Monocyte chemoattractant protein-1-induced tissue inflammation is critical for the development of renal injury but not type 2 diabetes in obese db/db mice. Diabetologia 2007; 50:471-80.

3. Goldberg RB. Cytokine and cytokine-like inflammation markers, endothelial dysfunction, and imbalanced coagulation in development of diabetes and its complications. J Clin Endocrinol Metab 2009; 94:3171-82.

4. Astrup AS, Tarnow L, Pietraszek L, Schalkwijk CG, Stehouwer CD, Parving $\mathrm{H}-\mathrm{H}$, et al. Markers of endothelial dysfunction and inflammation in type 1 diabetic patients with or without diabetic nephropathy followed for 10 years. Diabetes Care 2008; 31:1170-6.

5. Lim AK, Tesch GH. Inflammation in diabetic nephropathy. Mediators Inflamm 2012; 2012:146-54.

6. Martin J, Trowbridge E, Salmon G, Plumb J. The biological significance of platelet volume: its relationship to bleeding time, platelet thromboxane B2 production and megakaryocyte nuclear DNA concentration. Thrombosis Res 1983; 32:443-60.

7. Smith RA, Bosonnet L, Raraty M, Sutton R, Neoptolemos JP, Campbell $\mathrm{F}$, et al. Preoperative platelet-lymphocyte ratio is an independent significant prognostic marker in resected pancreatic ductal adenocarcinoma. Am J Surg 2009; 197:466-72.

8. Kwon HC, Kim SH, Oh SY, Lee S, Lee JH, Choi HJ, et al. Clinical significance of preoperative neutrophil-lymphocyte versus platelet-lymphocyte ratio in patients with operable colorectal cancer. Biomarkers 2012; 17:216-22.

9. Turkmen K, Erdur FM, Ozcicek F, Ozcicek A, Akbas EM, Ozbicer A, et al. Platelet-to-lymphocyte ratio better predicts 
inflammation than neutrophil-to-lymphocyte ratio in end-stage renal disease patients. Hemodialysis Int 2013; 17:391-6.

10. Temiz A, Gazi E, Güngör Ö, Barutçu A, Altun B, Bekler A, et al. Platelet/lymphocyte ratio and risk of in-hospital mortality in patients with ST-elevated myocardial infarction. Med Sci Monitor 2014; 20:660.

11. Akbas EM, Demirtas L, Ozcicek A, Timuroglu A, Bakirci EM, Hamur $\mathrm{H}$, et al. Association of epicardial adipose tissue, neutrophil-to-lymphocyte ratio and platelet-to-lymphocyte ratio with diabetic nephropathy. Int J Clin Exp Med 2014; 7:1794.

12. Hudzik B, Szkodzinski J, Lekston A, Gierlotka M, Polonski L, Gasior M. Mean platelet volume-to-lymphocyte ratio: a novel marker of poor short-and long-term prognosis in patients with diabetes mellitus and acute myocardial infarction. $J$ Diabetes Complications. 2016; 30:1097-102.

13. Kurtul A, Acikgoz SK. Usefulness of mean platelet volume-tolymphocyte ratio for predicting angiographic no-reflow and short-term prognosis after primary percutaneous coronary intervention in patients with ST-segment elevation myocardial infarction. Am J Cardiol 2017; 120:534-41.

14. Le Tulzo Y, Pangault C, Gacouin A, Guilloux V, Tribut O, Amiot L, et al. Early circulating lymphocyte apoptosis in human septic shock is associated with poor outcome. Shock 2002; 18:487-94.

15. An X, Ding PR, Li YH, Wang FH, Shi YX, Wang ZQ, et al. Elevated neutrophil to lymphocyte ratio predicts survival in advanced pancreatic cancer. Biomarkers 2010; 15:516-22.
16. Oylumlu M, Yildiz A, Oylumlu M, Yüksel M, Polat N, Bilik MZ, et al. Platelet-to-lymphocyte ratio is a predictor of in-hospital mortality patients with acute coronary syndrome. Anatol $J$ Cardiol 2015; 15:277-83.

17. Khan $\mathrm{YH}$, Sarriff A, Adnan AS, Khan AH, Malhi TH, Jummaat F. Complications associated with end stage renal disease. J Coll Physicians Surg Pak 2015; 25:392.

18. Xue R, Gui D, Zheng L, Zhai R, Wang F, Wang N. Mechanistic insight and management of diabetic nephropathy: Recent progress and future perspective. J Diabetes Res 2017; 2017: 1839809.

19. Qamar A, Hayat A, Ahmad TM, Khan A, Hasnat MNU, Tahir S. Serum cystatin $C$ as an early diagnostic biomarker of diabetic kidney disease in type 2 diabetic patients. $J$ Coll Physicians Surg Pak 2018; 28:288-91.

20. Matsusaka T, Sandgren E, Shintani A, Kon V, Pastan I, Fogo AB, et al. Podocyte injury damages other podocytes. J Am Soc Nephrol 2011; 22:1275-85.

21. Mundel P, Reiser J. Proteinuria: an enzymatic disease of the podocyte? Kidney Int 2010; 77:571-80.

22. Carmen M, Navarro JF. Inflammation and diabetic nephropathy. Cur Diabetes Rep 2006; 6:463-8.

23. Navarro-González JF, Mora-Fernández C. The role of inflammatory cytokines in diabetic nephropathy. J Am Soc Nephrol 2008; 19:433-42. 